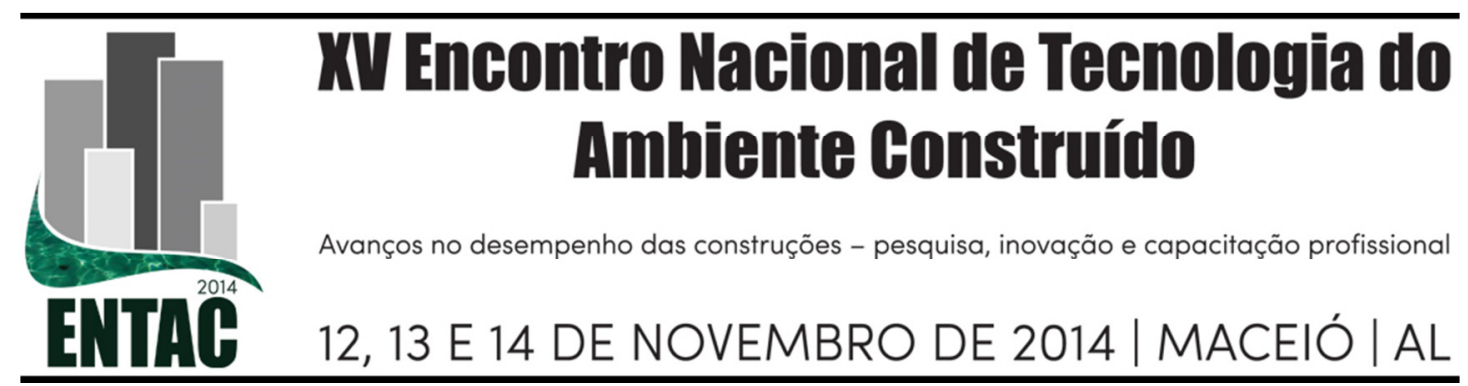

\title{
CARACTERIZAÇÃO E ANÁLISE DOS MÉTODOS DE ENTREGA DE PROJETO
}

\section{LINS, Deborah (1); CAMPOS, Ivna (2); MORORÓ, Mayra (3); BARROS NETO, José de Paula (4); CARDOSO, Daniel (5)}

(1) UFC, (85)86818584, deborahmo@gmailcom, (2)UFC, ivnabaquit@gmail.com, (3) UFC, mayrasoares.arq@gmail.com, (4) UFC, barrosneto@gercon.ufc.br, (5) UFC, daniel.br@mac.com

\begin{abstract}
RESUMO
Vários tipos de métodos de entrega de projeto estão atualmente disponíveis para os clientes, tais como Design-Bid-Build, Design-Build, Construction Management at Risk e IPD. Todos estes modelos já contribuíram para resultados de projetos com sucessos ou fracassos. É importante selecionar o modelo mais apropriado, pois as relações contratuais, leis e regulamentos vigentes, percepções de risco dos proprietários, mecanismos de concessão e a forma de pagamento podem influenciar na seleção de um ou outro modelo contratual. Assim sendo, o objetivo deste estudo é caracterizar e analisar os principais métodos de entrega de projeto utilizados na indústria da construção civil. Serão apresentadas informações relativas ao conceito, contextualização e análise comparativa entre os métodos pesquisados, a fim de orientar as partes envolvidas nos contratos na seleção e escolha do tipo mais adequado. O presente trabalho se insere no contexto do projeto de pesquisa em rede TICHIS / FINEP. O estudo do tipo descritivo é fruto de pesquisa bibliográfica e parte de uma discussão teórica que serviu de embasamento para os resultados parciais na referida pesquisa. Com apoio da revisão de literatura, pode-se comprovar que os princípios do IPD podem ser aplicados a qualquer modelo dentre os analisados. Porém, certas características de determinado projeto ou método de entrega podem afetar o nível de integração que se pretende alcançar. As contribuições residem no fato de que, a partir da análise realizada, é possível esclarecer os termos relativos a estes tipos de entrega de projeto, assim como colaborar com a literatura, uma vez que são escassas as pesquisas que abordam esse assunto, especialmente no Brasil.
\end{abstract}

Palavras-chave: tipos de contratação, métodos de entrega de projeto, Integrated Project Delivery.

\begin{abstract}
Various types of project delivery methods are currently available to customers, such as Design-Bid-Build, Design-Build, Construction Management at Risk and IPD. All these models have contributed to project outcomes with successes or failures. It is important to select the most appropriate model because contractual relations, laws and regulations, risk perceptions of the owners, granting mechanisms and the form of payment may influence the selection of one or other contractual model. Therefore, the aim of this study is to characterize and analyze key project delivery methods used in the construction industry. Information regarding the concept, context and comparative analysis of the methods surveyed are presented in order to guide the parties involved in contracts the selection and choose the most suitable type. This work is in the context of the research project on network TICHIS / FINEP. The descriptive study is the result of literature and part of a theoretical discussion that served as a basis for partial results in this research. With support from the literature review, one can prove that the principles of IPD can be applied to any model from the analysis. However, certain characteristics of a particular project or delivery method can affect the level of integration to be achieved. The contributions reside in the fact that, from the analysis, it is possible to clarify the terms for these types of project delivery, as well as collaborating with the literature, since there are few researches on this subject, especially in Brazil.
\end{abstract}

Keywords: types of contracting, project delivery, Integrated Project Delivery. 


\section{INTRODUÇÃO}

Vários tipos de métodos de entrega de projetos estão atualmente disponíveis para os clientes, e todos eles já contribuíram para resultados com sucessos e fracassos, especialmente nos Estados Unidos (TOURAN et al, 2009). Segundo os mesmos autores, é importante selecionar o modelo mais apropriado, pois as relações contratuais, leis e regulamentos vigentes, percepções de risco dos proprietários, mecanismos de concessão e a forma de pagamento podem influenciar na seleção de um método de entrega do projeto.

No gerenciamento tradicional de projetos selecionar o tipo de contrato mais apropriado, muitas vezes chamado de "método de entrega de projeto" (MEP), é entendido como uma das decisões mais importantes para o sucesso do projeto (MOSTAFAVI e KARAMOUZ, 2010).

Segundo Alarcón et al (2013), as três formas básicas de entrega de projeto possuem suas atividades com base no método do caminho crítico. Todas estas formas tradicionais de MEP se apoiam numa estratégia de fragmentação para o sucesso: otimizar cada pedaço para otimizar o projeto. Na prática, isto significa contratar cada etapa a um custo menor e usar o método do caminho crítico para gerenciar a sequência de atividades dependentes. Esta é a motivação principal: pressionar os participantes do caminho crítico para finalizar suas atividades dentro do prazo e do custo estabelecido (ALARCÓN et al, 2013).

Apesar desta lógica do gerenciamento tradicional de projetos, os proprietários e clientes continuam insatisfeitos: os empreendimentos são arriscados, demoram mais que o previsto, custam mais que o planejado e o produto final ainda não corresponde às expectativas dos clientes (LICHTIG, 2006). Na prática tradicional estes problemas surgem por conta da inadequada comunicação, cooperação, coordenação e integração entre as partes interessadas.

De acordo com Alarcón et al (2013), as características ou fatores chave que definem os MEP são estruturadas a partir de duas questões:

- Qual o acordo contratual entre os principais participantes do projeto (proprietário, projetistas e construtor)?

- O custo total da construção é um dos critérios de seleção final do construtor?

Ainda segundo os mesmo autores, as respostas se dividem em duas categorias: termos comerciais (incluindo seleção, compensação, riscos, responsabilidade e alocação); e organização para protocolos de comunicação e hierarquia.

Assim sendo, o objetivo desta pesquisa é caracterizar e analisar os principais MEP utilizados na indústria da construção civil. São apresentadas informações relativas ao conceito, contextualização e análise comparativa entre os métodos pesquisados, a fim de orientar as partes envolvidas nos contratos e a seleção e escolha do tipo mais adequado a cada caso.

O presente trabalho se insere no contexto do projeto de pesquisa em rede ${ }^{1}$ intitulado “Tecnologias da Informação e Comunicação aplicadas à Construção de Habitação de

\footnotetext{
${ }^{1}$ A rede de pesquisa congrega sete universidades brasileiras, quais sejam: Universidade de São Paulo USP; Universidade Federal do Rio Grande do Sul - UFRGS; Universidade Federal do Paraná - UFPR; Universidade Estadual de Campinas - UNICAMP; Universidade Presbiteriana Mackenzie - UPM; Universidade Federal da Bahia - UFBA; e Universidade Federal do Ceará - UFC.
} 
Interesse Social - TICHIS", financiado com recursos da Financiadora de Estudos e Pesquisas - FINEP. Tal projeto busca estimular o desenvolvimento tecnológico nessa área e tem como objetivo o desenvolvimento de soluções inovadoras em Tecnologia da Informação e Comunicação aplicadas à construção e arquitetura, visando a melhoria da qualidade e produtividade do segmento de HIS, com destaque para: BIM e outras soluções para suporte ao processo de gerenciamento de projetos, simulação de desempenho e operação de edificações.

Dessa forma, a pesquisa desenvolvida pela UFC pretendeu contribuir na execução de uma das metas da rede supracitada, através do desenvolvimento de dissertação de mestrado relacionada à gestão integrada de empreendimentos, estudando a inserção do IPD em HIS. O presente estudo é do tipo descritivo e fruto da pesquisa bibliográfica da referida dissertação, partindo de uma discussão teórica que serviu de embasamento para os resultados parciais do projeto TICHIS - FINEP. A metodologia detalhada da pesquisa completa pode ser auferida em Lins (2013).

\section{MÉTODOS TRADICIONAIS DE ENTREGA DE PROJETO}

Eastman et al (2008), apesar de reconhecer a existência de uma infinidade de modelos, considera que predominam três métodos fundamentais de entrega do projeto: DesignBid-Build (DBB), Design-Build (DB) e Construction Management at Risk (CMR). Esses modelos encontram correspondência, respectivamente, com os seguintes modelos tradicionalmente adotados no Brasil: Licitação pelo menor preço; Preço fixo e Administração da obra (UECHI et al, 2013). O desenvolvimento desta seção assim como a descrição a seguir dos tipos de MEP foram retirados de Lins (2013), Touran et al (2009) e AIA (2007).

\subsection{Design-Bid-Build (DBB):}

É o método tradicional de entrega de projeto. Neste método, o cliente, baseado em seus requisitos, convida um arquiteto ou projetistas para fornecer os serviços completos relativos ao projeto. Em seguida, este é colocado em "licitação" ou "concorrência", permitindo que o proprietário selecione um construtor, baseado no menor preço dado àquele conjunto de documentos relativos à construção. $\mathrm{O}$ cliente é o responsável pelos detalhes do projeto e garante a qualidade deste para a construtora.

No método de entrega DBB, o proprietário posiciona-se diretamente entre os projetistas e o construtor (Figura 01). Neste caso, ele é o "dono" do projeto durante a execução do empreendimento e é, por consequência, financeiramente responsável pelos custos de eventuais erros de projeto ou omissões da construção.

A fase de projetos DBB geralmente é atribuída por baixa oferta. Não há incentivo para o construtor minimizar os custos de alterações projetuais nesse tipo de método de entrega. $\mathrm{Na}$ verdade, pode acontecer exatamente o efeito contrário: um construtor que tenha vencido a "concorrência" com o menor preço, precisará procurar mudanças no projeto a fim de aumentar seu lucro no empreendimento.

Assim sendo, são características definidoras do DBB:

- Contratos separados para o projeto e para a construção;

- A escolha do construtor é baseada inteiramente no custo; e

- Os documentos relativos aos projetos estão $100 \%$ concluídos antes da definição do construtor. 
Como resultado, este modelo de entrega de projeto tem gerado uma quantidade significativa de problemas de construtibilidade e de coordenação que não são descobertos e resolvidos previamente na execução do empreendimento. Justamente por não possibilitar o envolvimento precoce do construtor no processo de projeto, este método de entrega oferece raras oportunidades para uma verdadeira integração dos agentes.

\subsection{Design-Build (DB):}

É um método de entrega de projeto em que o cliente adquire os serviços de projeto e construção num mesmo contrato através de um único representante legal, também conhecido como o construtor do projeto (empreiteiro geral). Para a escolha deste, existem várias maneiras, porém as mais comuns são realizadas em uma ou duas etapas, que podem ser realizadas simultaneamente ou uma a uma. Estas fases consistem de uma avaliação técnica (comprovação de qualificações e desempenho) e financeira (análise da proposta comercial). Dessa forma, no DB o construtor do projeto passa a ser o responsável por todos os custos do projeto e construção, uma vez que fornece o preço fixo por sua proposta.

O cliente muitas vezes escolhe o DB para transferir os riscos e os esforços de coordenação para um único contratado, a fim de garantir um maior nível de gerenciamento. Para isto, o proprietário teve seu envolvimento bem mais intenso no início do processo, estabelecendo critérios e definindo os requisitos do projeto a serem transmitidos ao construtor.

Do ponto de vista do proprietário, este modelo de entrega simplifica consideravelmente sua carga de responsabilidade no projeto (Figura 01). O construtor pode interferir com questões relativas à construtibilidade desde a fase de concepção. Como o cliente não participa dos detalhes do projeto, sua relação com o contratado deve ser baseada em um forte grau de confiança mútua, uma vez que detém o total controle deste método de entrega. Como resultado, este modelo tem sido muito bem sucedido especialmente no cumprimento dos prazos preestabelecidos (BEARD et al apud TOURAN et al, 2009).

Assim sendo, as características definidoras do DB são:

- Um único ponto de responsabilidade;

- Programação que permite a sobreposição do projeto e construção;

- Um construtor que fornece serviços preliminares ${ }^{2}$ de obra durante o desenvolvimento do projeto; e

- Um proprietário que espera do construtor do projeto um orçamento fixo e preciso, e que se comprometa com o cronograma de entrega.

Esta última característica, revela dois pontos de vista diferentes: se por um lado o cliente diminui os riscos transferindo responsabilidades ao construtor contratado, por outro lado, as oportunidades de melhorias e inovações no projeto são minimizadas; mesmo que o proprietário acompanhe todo o processo do empreendimento, sua interferência durante a execução é evitada.

Este modelo de entrega cria uma maior restrição à concorrência na medida em que as partes do contrato são selecionadas com base, principalmente, em sua qualificação e

\footnotetext{
${ }^{2}$ Os serviços preliminares aqui destacados podem incluir a orçamentação, estimativa de custos, estudos de viabilidade, programação, consultoria de construtibilidade e estudos de engenharia de valor, por exemplo.
} 
desempenho. Como o proprietário passa a responsabilidade para o construtor do projeto, ele acaba transferindo também a escolha dos subcontratados.

O método DB é contratualmente mais adaptado para aumentar a colaboração entre os membros da equipe de projeto-construção, apresentando assim, um ambiente propício para a implementação dos princípios do Integrated Project Delivery (IPD). Além disso, os principais participantes têm autonomia para escolher os outros membros da equipe e estabelecer suas próprias metodologias de trabalho.

\subsection{Construction Management ${ }^{3}$ at Risk (CMR):}

É um método de entrega de projeto caracterizado pelo contrato entre o proprietário e o gestor da construção, no qual assumem juntos os riscos relativos ao tempo e custo final do empreendimento. Neste tipo de acordo, o cliente dá autonomia para o construtor dar inputs durante o desenvolvimento do projeto. Entretanto, tradicionalmente o cliente acaba por assumir a responsabilidade de um contrato separado com o arquiteto.

A ideia do CMR é fornecer um gerenciamento profissional e de alto nível em todas as fases do ciclo de vida do projeto, uma vez que isoladamente não seriam capazes de realizar. Normalmente os contratos de CMR estipulam um Preço Máximo Garantido (PMG), acima do qual o proprietário não é responsável pelo pagamento. Muitas vezes, estes acordos incluem cláusulas de incentivo em que ambos podem dividir alguma economia de custos abaixo do PMG. Assim sendo, as características definidoras do CMR são:

- $\mathrm{O}$ arquiteto e o gestor da construção possuem contratos separados com o cliente;

- O gestor da construção é escolhido com base em outros critérios que não sejam apenas o menor custo, tais como a qualificação e desempenho em experiências passadas;

- O cronograma permite a sobreposição do projeto e construção;

- O proprietário usufrui dos serviços preliminares de obra do gestor da construção;

- O cliente espera que o gestor da construção cumpra o PMG e o cronograma de entrega do empreendimento;

- A transparência é aprimorada porque todos os custos e taxas são abertas e estão disponíveis, diminuindo as relações antagônicas entre os participantes do projeto.

A construtibilidade alcançada e a velocidade de implementação são os principais motivos para a escolha do CMR. Ao contrário do DBB, o CMR inclui o construtor no desenvolvimento do produto, podendo impactar positivamente na fase de projeto, tornando-se assim um membro colaborador da equipe.

O método CMR é apropriado tanto para projetos públicos como privados, em qualquer escala, quando o orçamento ou cronograma devem ser acompanhados de perto ou quando é requisitada uma coordenação extensiva dos consultores de projeto e parceiros comerciais. Como resultado, o CMR é um modelo de entrega particularmente adaptado ao IPD e com bastante potencial para possibilitar a entrega integrada de projetos. A Figura 01 representa a relação entre os agentes envolvidos nos três MEP.

\footnotetext{
${ }^{3}$ Construction Managament é um termo genérico aplicado a uma variedade de cenários possíveis de entrega de projeto nos quais os serviços de gestão são adicionados aos serviços gerais que um construtor geralmente oferece. Quando o gestor da construção e o construtor são a mesma pessoa, assume a responsabilidade de um empreiteiro geral, razão pela qual se acrescenta at Risk.
} 
Figura 01 - Esquema representativo dos métodos de entrega de projeto
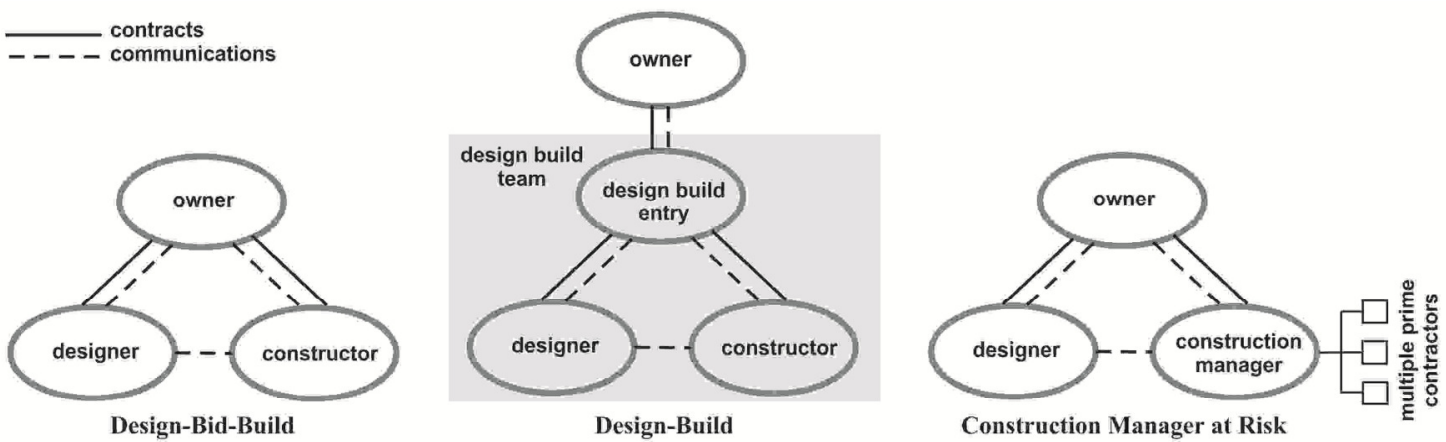

Fonte: AIA (2007)

Tradicionalmente a indústria da Arquitetura, Engenharia e Construção (AEC) têm adotado estes MEP, considerados convencionais. Entretanto, profissionais do setor estão insatisfeitos com os resultados dos projetos e argumentam que frequentemente os projetos ultrapassam a programação e o orçamento. Como um novo método de entrega de projeto, o IPD procura resolver os problemas de desperdício, ineficiência e de relações antagônicas dentro da indústria da AEC e aumentar a probabilidade de sucesso dos projetos (GHASSEMI e BECERIK-GERBER, 2011).

\section{INTEGRATED PROJECT DELIVERY}

\subsection{Definições e características:}

O termo Integrated Project Delivery foi desenvolvido pelo Institute of Architects (AIA), Califórnia Council. Em 2007, foi publicado o Integrated Project Delivery: a work definition para descrever o método de entrega de projeto do IPD. Em seguida, em parceria com o escritório nacional do AIA, foi publicado IPD: a guide. Além de apresentar definições e conceitos, esta publicação descreve como os métodos tradicionais de entrega do projeto podem ser beneficiados a partir da adoção de conceitos e ferramentas do método IPD (LINS, 2013).

O IPD é uma abordagem do processo de projeto que integra pessoas, sistemas, estruturas e práticas de negócio dentro de um processo que colaborativamente aproveita os talentos, conhecimentos e ideias de todos os participantes para otimizar os resultados do projeto, agregar valor ao cliente, reduzir desperdícios, e maximizar a eficiência de todas as fases do empreendimento: projeto, construção, uso e operação (AIA, 2007).

Foram desenvolvidos alguns documentos e tipos de contrato que facilitassem e estimulassem a colaboração entre os agentes envolvidos, dispositivo necessário em projetos de IPD. Apesar destes esforços, não existe uma definição padrão totalmente aceita pela indústria da construção como um todo. Diferentes definições, com níveis de sofisticação e abordagens muito variadas, apontam que o termo IPD é usado para descrever o processo de projeto da equipe e arranjos contratuais diferentes (SIVE, 2009). Segundo o AIA (2007), as principais características de um processo de projeto dentro da abordagem do IPD são:

- Processo altamente colaborativo, abrangendo desde a fase de concepção até entrega final do empreendimento;

- Equipe é guiada com base nos princípios de confiança e transparência; 
- Aproveita as contribuições e experiências dos participantes desde o início do processo, motivando seus membros a explorarem todo o seu potencial;

- Compartilhamento de informações entre os agentes envolvidos;

- Risco e recompensa dividida pelos membros da equipe (“o sucesso da equipe está ligado ao sucesso do projeto");

- Valor expandido do projeto, baseado na tomada de decisão coletiva;

- Utilização de recursos tecnológicos de apoio, resultando na oportunidade de projetar, construir e operar tão eficientemente quanto possível.

A estrutura de compensação do IPD segue o modelo do Project Alliancing, com o objetivo de estimular a eficiência e alinhar os interesses para o benefício do projeto como um todo. Neste modelo, os participantes do projeto são remunerados com base no "custo-plus", onde o proprietário garante o custo direto, porém uma parcela do lucro e bônus dos participantes são dependentes do resultado do projeto (AIA 2007).

Ao alinhar os objetivos das partes em torno do sucesso coletivo de um projeto e fazendo com que cada membro seja também responsável pelo comportamento dos outros, as equipes de projeto ganham mais controle do processo e diminuem o risco total (COHEN 2010).

Segundo Alarcón et al (2013), a nova estratégia para maximizar o desempenho do projeto é otimizar o projeto e não a etapa, conforme ilustrado na Figura 02.

Figura 02 - Abordagem tradicional versus Lean Project Delivery

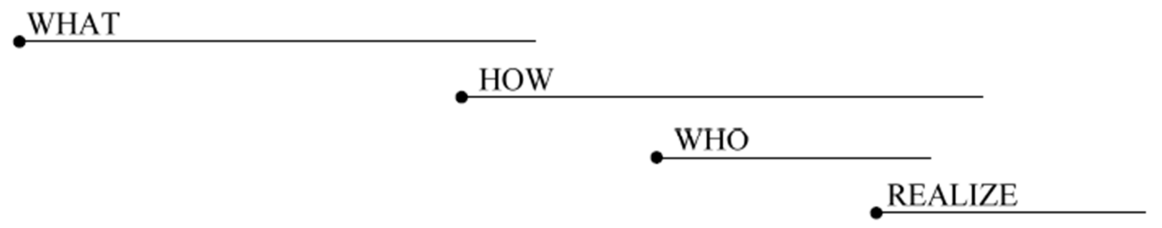

\begin{tabular}{|c|c|c|c|c|c|c|}
\hline Predesign & $\begin{array}{c}\text { Schematic } \\
\text { design }\end{array}$ & $\begin{array}{c}\text { Design } \\
\text { development }\end{array}$ & $\begin{array}{c}\text { Construction } \\
\text { documents }\end{array}$ & $\begin{array}{c}\text { Agency permit/ } \\
\text { Bidding }\end{array}$ & Construction & Closeout \\
\hline
\end{tabular}

TRADITIONAL

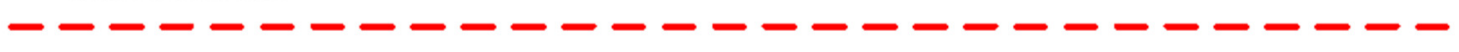

LEAN PROJECT DELIVERY

\begin{tabular}{|c|c|c|c|c|}
\hline $\begin{array}{c}\text { Project } \\
\text { definition }\end{array}$ & Lean design & Lean supply & Lean assembly & Use \\
\hline
\end{tabular}

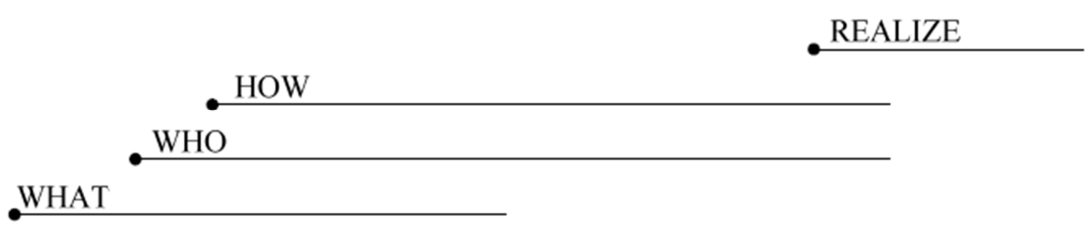

Fonte: Alarcón et al (2013)

Sob o ponto de vista do AIA (2007), no que diz respeito ao processo de projeto tradicional e ao processo de projeto integrado, estes diferem especialmente quanto às equipes de projeto, quanto ao processo, aos riscos, às compensações, tecnologias adotadas e acordos, conforme apresenta o Quadro 01: 
Quadro 01 - Processo de projeto tradicional x Processo de projeto IPD:

\begin{tabular}{|c|c|c|}
\cline { 2 - 3 } \multicolumn{1}{c|}{$\begin{array}{c}\text { EQUIPES DE } \\
\text { PROJETO }\end{array}$} & $\begin{array}{c}\text { Equipe fragmentada, hierarquizada, } \\
\text { controlada. Trabalho individual. Realiza } \\
\text { somente o básico e necessário. }\end{array}$ & $\begin{array}{c}\text { Equipe integrada, multidisciplinar, aberta e } \\
\text { colaborativa. Montada previamente e com } \\
\text { participantes chave }\end{array}$ \\
\hline $\begin{array}{c}\text { PROCESSO DE } \\
\text { PROJETO }\end{array}$ & $\begin{array}{c}\text { Linear, sequencial e segregado. } \\
\text { Conhecimentos adquiridos somente quando } \\
\text { necessário. Informações acumuladas de } \\
\text { outras experiências. }\end{array}$ & $\begin{array}{c}\text { Colaborativo, concorrente e multidisciplinar. } \\
\text { Conhecimentos adquiridos previamente. } \\
\text { Informações compartilhadas. }\end{array}$ \\
\hline RISCOS & $\begin{array}{c}\text { São geridos individualmente e transferidos } \\
\text { sempre que possível. }\end{array}$ & $\begin{array}{c}\text { São geridos coletivamente e assim também } \\
\text { compartilhados. }\end{array}$ \\
\hline $\begin{array}{c}\text { COMPENSAÇÕES E } \\
\text { RECOMPENSA }\end{array}$ & $\begin{array}{c}\text { Perseguidas individualmente. Procura-se o o } \\
\text { mínimo de esforço para o máximo de retorno. }\end{array}$ & $\begin{array}{c}\text { Baseado no valor agregado. O sucesso da } \\
\text { equipe é o sucesso do projeto. }\end{array}$ \\
\hline COMUNICAÇÃO & $\begin{array}{c}\text { É analógico, bidimensional, baseado no no } \\
\text { papel. Gera documentação duplicada, perda } \\
\text { de informação. Compatibilização entre as } \\
\text { disciplinas de projeto. }\end{array}$ & $\begin{array}{c}\text { Apresenta base digital, virtual. Possui banco } \\
\text { de dados. Baseado em modelos 3D, 4D e 5D. } \\
\text { Disciplinas integradas desde o início e } \\
\text { detecção automática de interferências. }\end{array}$ \\
\hline ACORDOS & $\begin{array}{c}\text { Contratos bilaterias. Incentiva esforços } \\
\text { unilaterais e transferência de riscos, sem } \\
\text { compartilhamento de informações }\end{array}$ & $\begin{array}{c}\text { Contratos multilaterais. Incentiva o apoio } \\
\text { horizontal entre os membros, riscos divididos, } \\
\text { com compartilhamento de informações }\end{array}$ \\
\hline
\end{tabular}

Fonte: elaborado pelos autores

\subsection{Questões contratuais}

Um dos principais objetivos do IPD é maximizar a colaboração e a coordenação com vistas à totalidade do projeto e seus resultados e, portanto, os contratos tornam-se elemento fundamental para alcançar o sucesso das metas estabelecidas coletivamente, $\mathrm{o}$ que seria muito complicado realizar através de contratos separados que criam motivações opostas (BECERIK-GERBER e KENT, 2010).

A estrutura contratual atual provoca conflitos e ineficiências, pois incentiva cada uma das partes a se preocupar com seus próprios interesses e não os interesses do projeto como um todo (AIA, 2007).

Dentro do contexto brasileiro, o nível de qualidade dos serviços prestados pelos fornecedores depende da relação pessoal com o construtor. As construtoras estrangeiras encontram dificuldades para contratar os fornecedores locais, devido a exigências rigorosas nos contratos. As relações técnicas e comerciais são marcadas pela informalidade, ao contrário de outros países, onde as relações profissionais são convencionadas por contratos específicos (LINS, 2013).

Segundo Matthews e Howell (2005), existem dois tipos de contratos: transacionais e relacionais. Os contratos transacionais são definidos por troca de bens e serviços. Já os contratos relacionais assemelham-se a pequenas sociedades, com seu próprio sistema interno de normas de envolvimento, que vão além daquelas centradas na troca de seus processos imediatos.

O IPD emprega ambos os contratos, transacionais e relacionais. Externamente, é utilizado um contrato transacional clássico com o cliente e os participantes chave. Internamente, todos os membros envolvidos estão vinculados por um contrato relacional, comumente descrito de "pacto", no qual todos assinam. O "pacto" minimiza os custos do transacional, ligando os membros da equipe em uma parceria enquanto durar o projeto (MATTHEWS e HOWELL, 2005).

Entretanto, os contratos de IPD são mais parecidos com os contratos relacionais porque "consideração" é colocada no processo e não apenas no produto final. Quando os 
membros de uma equipe têm alguma expectativa de trabalharem juntos novamente no futuro, eles abordam e gerenciam suas atividades tendo em mente uma relação de futuro pré-estabelecida (LINS, 2013).

Abordagens integradas envolvem relações contratuais que são bastante diferentes dos modelos tradicionais de contrato. Modificá-los com vistas à integração pode ser um desafio, porque as abordagens são muito diferentes entre si. Negociar e elaborar contratos sem o auxílio de um contrato similar anterior ou formulários padrão pode aumentar o custo de se chegar a um acordo (AIA, 2007).

\section{RESULTADOS E DISCUSSÃO}

As principais diferenças entre o IPD e outros métodos estão nos termos de contrato, nas relações entre os membros da equipe de projeto e nas estruturas de compensação (BECERIK-GERBER e KENT, 2010).

Em contrapartida, Wright (2012) adverte que ainda não está claro se o IPD deve ser considerado como um método de entrega de projeto ou como um contrato multilateral requisitado. A partir disso, o mesmo autor discute essa definição como uma operação em um dos três níveis de colaboração: nível 1 ou "típico", na qual a colaboração não está totalmente exigida; nível 2 ou "aprimorada", na qual alguma colaboração contratual é exigida; e nível 3 ou "exigida", na qual a colaboração é obrigatória, através de um contrato multilateral.

Dando continuidade a esta discussão, pode-se perceber que, apesar de cada método de projeto ser coerente em sua própria abordagem, podem misturar e combinar características. Por exemplo, é possível aplicar um fluxo de trabalho focado na organização colaborativa dentro de um contrato transacional.

O IPD pode ser mais precisamente definido e caracterizado não apenas como uma filosofia, mas como outro sistema de entrega do projeto. Além disso, outra característica importante é a maneira como alcançar as metas do projeto, sob uma atmosfera de colaboração e integração que é reforçada pelo uso de contrato relacional.

Segundo AIA (2007), os princípios do IPD podem ser aplicados a qualquer modelo de entrega de projeto. No entanto, certas características de determinado projeto ou modelo de entrega podem afetar o nível de integração que se pretende alcançar.

\section{CONSIDERAÇÕES FINAIS}

A partir das análises e comparações desenvolvidas ao longo do trabalho, percebe-se que os principais Métodos de Entrega de Projeto diferenciam-se entre si especialmente pelo arranjo contratual, estrutura de compensação e nível de integração entre seus participantes.

A definição prévia destes fatores chave pode caracterizar melhor o tipo de relação e envolvimento dos participantes do empreendimento, assim como interfere diretamente na maneira como o projeto é concebido e gerenciado. A partir da caracterização realizada, é possível escolher o Método de Entrega de Projeto mais indicado a cada caso.

Com apoio da revisão de literatura, pode-se comprovar que os princípios do IPD podem ser aplicados a qualquer modelo dentre os analisados. No entanto, certas características de determinado projeto ou método de entrega podem afetar o nível de integração que se pretende alcançar. 
As contribuições deste estudo residem no fato de que, a partir da análise realizada, é possível esclarecer os termos relativos a estes tipos de entrega de projeto, assim como colaborar com a literatura, uma vez que são escassas as pesquisas que abordam esse assunto, especialmente no Brasil.

\section{AGRADECIMENTOS}

Os autores agradecem ao CNPQ, pela concessão de bolsa de pesquisa.

\section{REFERENCIAS}

ALARCÓN, L. F.; MESA, H.; HOWELL, G. Characterization of lean project Delivery. In: IGLC, 21., 2013, Proceedings... Fortaleza, 2013.

AMERICAN INSTITUTE OF ARCHITECTS (AIA) and AIA CALIFORNIA COUNCIL. Integrated Project Delivery: A Guide. Version 1, 2007.

BECERIK-GERBER, B.; KENT, D.C. Implementation of Integrated Project Delivery and Building Information Modeling on a Small Commercial Project. Associated Schools of Construction Annual International, e CIB Workgroup 89, Massachusetts. Abril, 2010.

COHEN, J. Integrated Project Delivery: Case Studies. AIA, AIA CC, AGC Califórnia, MacGraw Hill Construction, 2010.

EASTMAN, C. et al. BIM Handbook: a guide to building information modeling to owners, managers, designers, engineers, and contractors. New Jersey: John Wiley \& Sons, 2008.

GHASSEMI, R., BECERIK-GERBER B. Transitioning to Integrated Project Delivery: Potential Barriers and Lessons Learned. Lean Construction Journal. 2011, p. 32-52.

LICHTIG, W. A. The integrated agreement for lean project delivery. Construction Lawyer, 2006, V.26, n 3

LINS, D. M. O. Integrated Project Delivery: diretrizes para empresas de projeto que atuam em habitação de interesse social. 2013. Dissertação (Mestrado em Engenharia Civil) - Centro de Tecnologia da Universidade Federal do Ceará, Fortaleza, 2013.

MATTHEWS, O.; HOWELL, G. A. Integrated Project Delivery. An example of relational contracting. Lean Construction Journal, 2005, V. 2, p. 42-61, April/ 2005.

MOSTAFAVI, A.; KARAMOUZ, M. Selecting appropriate project delivery system: Fuzzy approach with risk analysis. ASCE, 2010.

SIVE, T. Integrated Project Delivery: reality and promise. A strategist's guide to understanding and marketing IPD. Society for Maketing Professional Services Foundation White Paper on IPD, Smps Publication 21, 2009.

TOURAN, A.; GRANSBERG, D. D.; MOLENAAR, K. R.; GHAVAMIFAR, K.; MASON, D. J.; FITHIAN, L. A. TCRP Report 131: A Guidebook for the Evaluation of Project Delivery Methods. Transportation Research Board. Washignton D.C., 2009.

UECHI, M. E.; PAULA, N. de; MOURA, N. C. da S. Projeto Integrado de edifícios - análise da postura dos profissionais. In: SBQP, 3.; TIC, 6., 2013, Campinas. Anais... Porto Alegre: ANTAC, 2013.

WRIGHT, J. A. The integration of building information modeling and integrated project delivery into the construction management curriculum. In: AMERICAN SOCIETY FOR ENGINEERING EDUCATION ANNUAL CONFERENCE, 2012. 